\title{
Correlation of serum neutrophil gelatinase- associated lipocalin with acute kidney injury in hypertensive disorders of pregnancy
}

This article was published in the following Dove Press journal: International Journal of Nephrology and Renovascular Disease I October 2013

Number of times this article has been viewed

\author{
ML Patel' \\ Rekha Sachan ${ }^{2}$ \\ Radheyshyam Gangwar ${ }^{3}$ \\ Pushpalata Sachan ${ }^{4}$ \\ SM Natu \\ 'Department of Medicine, King \\ George's Medical University, Lucknow, \\ Uttar Pradesh, India; ${ }^{2}$ Department \\ of Obstetrics and Gynaecology, King \\ George's Medical University, Lucknow, \\ Uttar Pradesh, India; ${ }^{3}$ Department \\ of Critical Care, Sanjay Gandhi \\ Post Graduate Institute of Medical \\ Sciences, Lucknow, Uttar Pradesh, \\ India; ${ }^{4}$ Department of Physiology, King \\ George's Medical University, Lucknow, \\ Uttar Pradesh, India; ${ }^{5}$ Department \\ of Pathology, King George's Medical \\ University, Lucknow, Uttar Pradesh, \\ India
}

\begin{abstract}
Hypertensive disorders of pregnancy (HDP) remain one of the largest single causes of maternal and fetal morbidity and mortality, accounting for $16.1 \%$ of maternal deaths in developed countries. The aim of the study was to evaluate acute kidney injury (AKI) in hypertensive disorders of pregnancy and to examine the correlation of serum neutrophil gelatinase-associated lipocalin (NGAL) with acute kidney injury. This prospective case control study was carried out over a period of 1 year. After written, informed consent and ethical clearance, 149 cases of hypertensive disorders of pregnancy were screened, and seven were lost to follow-up. Acute kidney injury was detected in 88 cases and acute renal failure in 30 cases of HDP. Thirty-one healthy pregnant nonhypertensive women were enrolled as controls. Quantitative measurement of serum NGAL levels was done by enzyme linked immunosorbent assay technique using a sandwich enzyme-linked immunosorbent assay kit. As per the Kidney Diseases Improving Global Outcomes International guidelines acute kidney injury network (AKIN), 50 cases $(42.37 \%)$ of AKI stage I, 38 (32.2\%) cases of AKI stage II, and 30 (25.42\%) cases of renal failure were detected. Serum NGAL had a positive association with increasing proteinuria. It also had a positive correlation with systolic blood pressure $(r \sim 0.36)$, diastolic blood pressure $(r \sim 0.37)$, and serum creatinine $(r \sim 0.4)$. NGAL was found to be significantly correlated with creatinine in the cases with the value of the correlation coefficient being 0.4 . This direct correlation might be a consequence of endothelial dysfunction on which hypertension and proteinuria probably depends.
\end{abstract}

Keywords: hypertensive disorders of pregnancy, preeclampsia, eclampsia, serum neutrophil gelatinase-associated lipocalin, acute kidney injury

\section{Introduction}

Hypertensive disorders of pregnancy (HDP) remains one of the largest single causes of maternal and fetal morbidity and mortality, accounting for maternal death in developed countries, Latin America, and Africa at 16.1\%, 25.7\%, and 9.1\%, respectively, and $18 \%$ of fetal deaths are associated with hypertensive disorders. ${ }^{1,2}$ The pathogenesis of HDP is still a matter of debate. It is a multifactorial disease and its pathogenesis involves a series of complex mechanisms including incomplete invasion and remodeling of the maternal spiral arteries, high circulating level of antiangiogenic factors, proinflammatory cytokines, and generalized endothelial dysfunction. The neutrophil gelatinase-associated lipocalin (NGAL) concentrations are low in healthy human tissues (such as kidney, lung, stomach, liver, and colon), but appear to be upregulated in pathological conditions following endothelial cell injury. ${ }^{3}$ In a multicenter study, approximately $30 \%$ of HDP cases were due to chronic hypertension, while $70 \%$ of cases were due to gestational hypertension/preeclampsia. ${ }^{4}$
Correspondence: ML Patel Department of Medicine, King George's Medical University, Lucknow-226003,

Uttar Pradesh, India

Tel +9l 09839007000

Email apatelsac@gmail.com 
A commonly used marker for kidney injury is serum creatinine but it is slow and insensitive and is not able to be detected in subtle injuries. Serum creatinine requires several hours to days to accumulate. It increases in serum only after $50 \%$ or more of renal function is $\operatorname{lost}^{5}$ and its concentration is affected by multiple confounding factors. Effective preventive and therapeutic measures are available, but diagnosis of acute kidney injury (AKI) in HDP is frequently delayed due to lack of an early predictive biomarker. One of the promising biomarkers, NGAL is a $25 \mathrm{kDa}$ member of the lipocalin family that is highly accumulated in blood after nephrotoxic and ischemic renal insult. ${ }^{6}$ After ischemic injury, NGAL levels in kidney tissue rise by 10 -fold within 3 hours. ${ }^{7}$ This rise appears to be sustained, being evident for several days following the initial insult. This pattern of early rise and persistence makes NGAL a highly sensitive marker for diagnosing AKI. There is no study which assesses AKI in HDP in very early stages. Therefore, this study was planned and the aim was to evaluate AKI in HDP and to examine the association of serum NGAL with AKI in these subjects.

\section{Material and methodology}

This prospective case control study was carried out over a period of 1 year at King George's Medical University, Lucknow, Uttar Pradesh, India in collaboration with the Department of Internal Medicine and Department of Obstetrics and Gynaecology. After written, informed consent and ethical clearance from the institutional ethics committee, 149 cases of HDP defined as per the National High Blood Pressure Education Program Working Group $(2000)^{8}$ were screened for AKI. Seven cases were lost to follow-up. Out of 142 cases of HPD, AKI was detected in 88 cases and acute renal failure in 30 cases. Thirty-one healthy pregnant nonhypertensive women were taken as controls. The women enrolled had a gestational age of 20-40 weeks. Grades of AKI were defined according to Kidney Diseases Improving Global Outcomes International Guidelines group (KDIGO) 2011 guidelines acute kidney injury network (AKIN). ${ }^{9}$

Women suffering with chronic kidney disease, chronic hypertension, chronic liver disease, cardiovascular disease, or who had infective and neoplastic pathology were excluded from the study. Data were collected and analyzed. Demographic characteristics, blood pressure on admission, biochemical parameters including serum urea, serum creatinine, liver function test, serum uric acid, serum lactic dehydrogenase, and 24 hour urinary protein excretion were recorded. All patients were assessed by the obstetrician for maternal and fetal well-being.
Measurement of blood pressure was done 4 hourly, in the right arm in the supine position. Korotkoff V sounds were taken for measurement of diastolic blood pressure by a mercury sphygmomanometer.

Mild preeclampsia was defined as blood pressure $\geq 140 / 90 \mathrm{mmHg}$ but less than $160 / 110 \mathrm{mmHg}$ with proteinuria $\geq 300 \mathrm{mg} / 24$ hours. Severe preeclampsia was defined as blood pressure $\geq 160 / 110 \mathrm{mmHg}$ with urinary protein excretion of $\geq 2.0 \mathrm{~g} / 24$ hours or any of the following symptoms: oliguria or $<400 \mathrm{~mL}$ urine/ 24 hours, visual disturbances, serum creatinine $\geq 1.2 \mathrm{mg} / \mathrm{dL}$, platelets less than $100,000 / \mathrm{mm}^{3}$, or microangiopathic hemolysis. Eclampsia was defined as occurrence of new onset grand mal seizure in a patient with preeclampsia.

Five milliliters of venous blood was obtained in a plain vial from the cases and controls. Collected blood samples were stored at $4^{\circ} \mathrm{C}$. Centrifugation was done at $6000 \mathrm{rpm}$. Samples were frozen at $-20^{\circ} \mathrm{C}$ until assayed. Quantitative measurement of serum NGAL levels was done by enzyme-linked immunosorbent assay by using a sandwich enzyme-linked immunosorbent assay kit (USCN Life Science Inc, Wuhan, Hubei, People's Republic of China). All relevant investigations were carried out in an accredited laboratory (Department of Pathology, King George's Medical University, Lucknow, Uttar Pradesh, India). Estimation of serum creatinine was done by Jaffe Reaction, using Technicon AutoAnalyzer methodology (Technicon Instrument Corp., Chauncy, NY, USA) 1963:N-11a and uric acid estimation was done by nephelometry. All patients were followed until they were discharged from the hospital.

\section{Statistical analysis}

Data were analyzed using the statistical software package STATA (version 11.2, College Station, TX, USA). A difference between the two values was considered to be significant if the $P$-value was $\leq 0.05$. The categorical data were described as a percentage and continuous variables as mean \pm standard deviation. The association between two or more categorical variables was tested by $\chi^{2}$ statistics by using appropriate correction. Prior to carrying out any test on continuous data, the normalcy of data was tested. The twosample $t$-test was used to see the difference between the mean of two different groups if data were normally distributed. If data were not found to be normally distributed, the MannWhitney test was used to test the level of significance between two values. One-way analysis of variance was used to test the difference among $>2$ groups in case of normally distributed data. Bonferroni correction was applied to the 
level of significance to avoid family error. If the data were not normally distributed, the Kruskal-Wallis test was used in place of one-way analysis of variance.

\section{Results}

Analysis was performed on 118 cases of AKI and 31 healthy pregnant nonhypertensive controls. Table 1 shows that the maximum number of cases 77 (65.25\%) and controls $22(70.97 \%)$ were in the age range of $25-35$ years. ${ }^{10}$ The majority of the controls $27(87.10 \%)$ belonged to a middle socioeconomic status and a maximum of 63 (53.38\%) cases also belonged to middle class (based on Kuppu Swamy's Socioeconomic status scale updated 2012). ${ }^{10}$ The mean period of gestational age was almost equal in both groups. Sixty-three $(53.38 \%)$ cases were primigravida and 55 $(46.61 \%)$ cases were multigravida, whereas 13 controls $(41.94 \%)$ were primigravida. None of the cases or controls had a history of similar disease in the past (Table 1).

The mean systolic blood pressure among controls was $117.84 \pm 4.7 \mathrm{mmHg}$, and in the cases it was $152.61 \pm 9.58 \mathrm{mmHg}$. The mean diastolic blood pressure in the controls was $77.42 \pm 6.8 \mathrm{mmHg}$, whereas in the cases it was $99.07 \pm 7.36 \mathrm{mmHg}$. A statistically significant difference was observed between the mean values of systolic blood pressure and diastolic blood pressure among cases and controls $(P<0.0001)$.

Table I Demographic profile

\begin{tabular}{|c|c|c|}
\hline Groups & $\begin{array}{l}\text { Control }(n=3 I) \\
n(\%)\end{array}$ & $\begin{array}{l}\text { AKI + ARF }(n=118) \\
n(\%)\end{array}$ \\
\hline \multicolumn{3}{|l|}{ Age groups } \\
\hline$<25$ years & $9(29.03)$ & $4 \mid(34.75)$ \\
\hline $25-35$ years & $22(70.97)$ & $77(65.25)$ \\
\hline \multicolumn{3}{|l|}{ Gravida } \\
\hline 1 & $13(4 \mid .94)$ & $63(53.38)$ \\
\hline$\geq 2$ & $18(58.06)$ & $55(46.61)$ \\
\hline Mean gestational age & $35.86 \pm 2.0$ & $35.80 \pm 3.6$ \\
\hline \multicolumn{3}{|l|}{ Socioeconomic status } \\
\hline Low & $4(12.90)$ & $55(46.6 \mathrm{I}) * * *$ \\
\hline Medium & $27(87.10)$ & $63(53.38)$ \\
\hline \multicolumn{3}{|c|}{ Past history of hypertensive disease in pregnancy } \\
\hline Not present & $31(100)$ & $118(100)$ \\
\hline Present & $0.0(0.0)$ & $0.0(0.0)$ \\
\hline \multicolumn{3}{|l|}{ CNS } \\
\hline Normal & $100(31)$ & I0I (85.59) \\
\hline Abnormal & $0.00(0)$ & $17(14.4 I)$ \\
\hline \multicolumn{3}{|l|}{ Hypertension } \\
\hline Mean SBP $(\mathrm{mmHg})$ & $117.84 \pm 4.7$ & $|52.6| \pm 9.58 * * *$ \\
\hline Mean DBP $(\mathrm{mmHg})$ & $77.42 \pm 6.8$ & $99.07 \pm 7.36 * * *$ \\
\hline
\end{tabular}

Notes: ***Denotes a significant difference is observed between the mean values of systolic and diastolic blood pressure among controls and cases $(P<0.00 \mathrm{I})$. Controls do not match the cases in terms of socioeconomic status.

Abbreviations: AKI, acute renal injury; ARF, acute renal failure; DBP, diastolic blood pressure; SBP, systolic blood pressure; CNS, central nervous system.
Out of 142 cases of HPD, AKI was detected in 88 cases and acute renal failure in 30 cases as per the KDIGO guidelines (AKIN). ${ }^{9}$ Fifty cases $(42.37 \%)$ of AKI stage I, 38 (32.2\%) cases of AKI stage II, and $30(25.42 \%)$ cases of acute renal failure were detected. After analysis within the groups (AKI stages I and II and acute renal failure), it was observed that the mean serum NGAL level was lowest in the control group and highest in the acute renal failure group (Figure 1).

Figure 2 shows correlation coefficient of serum NGAL with various parameters. When we pooled data, serum NGAL had a positive correlation with systolic blood pressure $(r \sim 0.36)$, diastolic blood pressure $(r \sim 0.37)$, and with serum creatinine $(r \sim 0.4)$. It also had a positive correlation with serum lactate dehydrogenase $(\mathrm{LDH})(r \sim 0.32)$, serum bilirubin ( $r \sim 0.32)$, and liver enzymes. Serum NGAL had a negative correlation with the birth weight of the baby and with urine output (Table 2).

The mean serum NGAL level in controls without proteinuria was $294.61 \pm 356.2 \mathrm{pg} / \mathrm{mL}$. The maximum mean serum NGAL level was observed when proteinuria was between 3 and $5 \mathrm{~g} / 24$ hours with all grades of AKI and acute renal failure. This finding suggests that serum NGAL has a positive association with increasing proteinuria. The difference between the mean serum NGAL levels between cases and controls was statistically significant in all grades of AKI and acute renal failure $(P<0.0001)$ (Table 2$)$.

Within the cases (pooled data), there was a significant positive correlation between the values of serum NGAL and serum creatinine $\left(\mathrm{y}=1.56+0.00007^{*} \mathrm{x}, r=0.4\right)(P<0.01)$ (Figure 3$)$. In the control group, there was a negative correlation between serum NGAL and serum creatinine $(\mathrm{y}=0.6-0.00003 \mathrm{x}, r=0.06)$ (Figures 3 and 4).

Table 2 Association between proteinuria and serum neutrophil gelatinase-associated lipocalin levels

\begin{tabular}{llll}
\hline Proteinuria & $\begin{array}{l}\text { AKI stage I } \\
\text { S. NGAL } \\
(\mathbf{p g} / \mathbf{m L})\end{array}$ & $\begin{array}{l}\text { AKI stage II } \\
\text { S. NGAL } \\
(\mathbf{p g} / \mathbf{m L})\end{array}$ & $\begin{array}{l}\text { ARF } \\
\text { S. NGAL } \\
(\mathbf{p g} / \mathbf{m L})\end{array}$ \\
\hline Nil & $294.61 \pm$ & $294.61 \pm$ & $294.61 \pm$ \\
& 356.20 & 356.20 & 356.20 \\
$\geq 0.3$ g/24 hours & $3799.88 \pm$ & $4821.82 \pm$ & $1245.0 \pm$ \\
& 3504.37 & 4465.93 & 0.0 \\
$\geq 2$ g/24 hours & $4844.19 \pm$ & $11176.63 \pm$ & $10798.79 \pm$ \\
& 5137.57 & 3761.05 & 2916.01 \\
$\geq 3-5$ g/24 hours & $11343.0 \pm$ & $12175.95 \pm$ & $12106.38 \pm$ \\
& 3316.24 & 2944.55 & 3325.28 \\
P-value & $<0.001$ & $<0.001$ & $<0.001$ \\
\hline
\end{tabular}

Note: The comparisons between the different categories have been done using the Kruskal-Wallis test (nonparametric analog of analysis of variance).

Abbreviations: AKI, acute kidney injury; ARF, acute renal failure; S. NGAL, serum neutrophil gelatinase-associated lipocalin. 


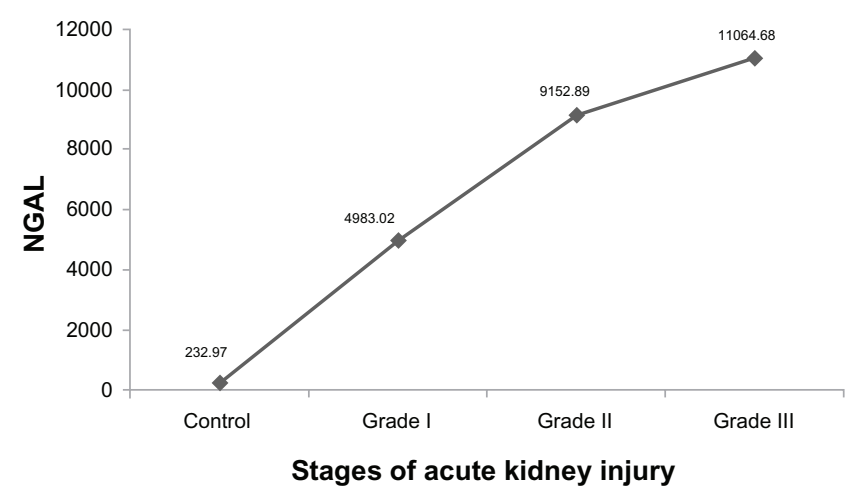

Figure I Association between mean values of serum neutrophil gelatinaseassociated lipocalin with various grades of acute kidney injury.

Note: Grade III taken as acute renal failure.

Abbreviation: NGAL, neutrophil gelatinase-associated lipocalin.

\section{Discussion}

Immediately after AKI, blood urea and serum creatinine may be normal and the only sign may be decreased urine output. ${ }^{11}$ At the same time, the degree of oliguria does not necessarily correlate with the severity of injury. AKI can occur without affecting urine output, which is classified as oliguric or nonoliguric, and it has a prognostic value rather than a diagnostic indication. ${ }^{12}$ A commonly used marker for kidney injury is serum creatinine but it is slow, insensitive, and is not able to detect subtle injuries. Markers that help to identify kidney injury earlier than changes in serum creatinine are needed that can predict clinical outcomes and efficacy of therapy, and where the results are available while damage is reversible. ${ }^{10}$ Effective, preventive, and therapeutic measures are available, but diagnosis of AKI is frequently delayed due to lack of early predictive biomarkers. One promising candidate is NGAL that is highly accumulated in blood and urine after nephrotoxic and ischemic insult.

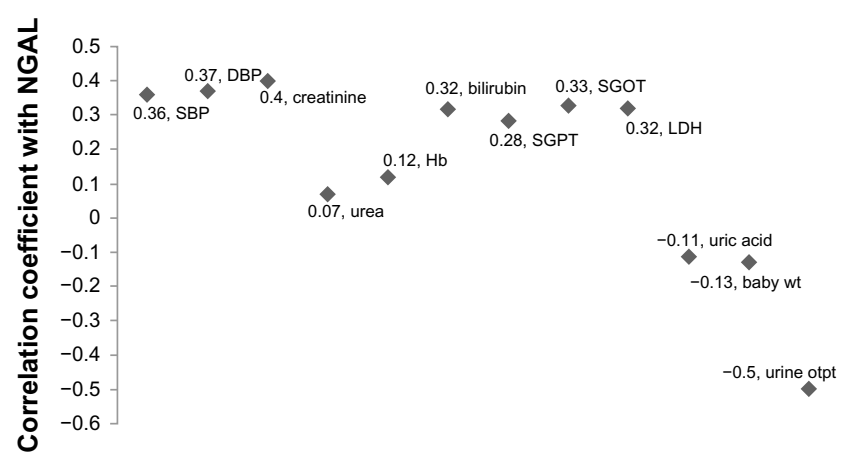

Biochemical parameters

Figure 2 Scatter diagram showing correlation coefficients of neutrophil gelatinaseassociated lipocalin with various parameters in pooled data.

Abbreviations: DBP, diastolic blood pressure; LDH, lactate dehydrogenase; SBP, systolic blood pressure; Hb, hemoglobin; SGPT, serum glutamic pyruvic transaminase; SGOT, serum glutamic oxaloacetic transaminase; Baby wt, baby weight, Urine otpt, urine output; NGAL, neutrophil gelatinase-associated lipocalin.
In our study, the mean serum NGAL levels were significantly increasing with increasing grades of AKI, and almost two- to five-fold increase in serum NGAL was found. Acute renal failure was found in $25.42 \%$ cases of HDP according to the definition of KDIGO 2011 (AKIN), ${ }^{9}$ where as others ${ }^{13}$ reported AKI in $20.76 \% .{ }^{13}$ Usually, studies report AKI in HDP when patients are referred with diagnosis of acute renal failure needing renal replacement therapy (AKI Stage III/acute renal failure). In our study, AKI stage I was found in $42.37 \%$ of cases and AKI stage II in $32.2 \%$ of cases. Similarly, an Indian study reported pregnancy related acute kidney injury in preeclampsia 30.5\%. This high incidence of $\mathrm{AKI}$ in the $\mathrm{HDP}^{14}$ group may be due to late referral of cases when renal damage has already occurred. In our study, there were $30(25.42 \%)$ cases of acute renal failure, not only due to HDP. These cases also had other complications like abruption (13 cases), postpartum hemorrhage (four cases), septicemia (eleven cases), jaundice (one case), and pancreatitis (one case). Acute kidney injury stages I and II are often missed, so are often not reported, and the patient is not referred until a late stage (ARF when renal replacement therapy is required). However, a previous study shows that NGAL failed to reliably predict changes in serum creatinine, even though its predictive value improved with increasing AKI severity. ${ }^{15}$

HDP comprises preeclampsia and eclampsia. The former is a multisystem disorder of unknown etiology that affects $4 \%-5 \%$ of pregnancies. ${ }^{16,17}$ The incidence of eclampsia is $0.3 \%-9 \%$, and it has a maternal mortality rate of $0.5 \%-10 \%{ }^{18}$ In our study, other findings of note were the positive correlation between serum NGAL and covariates, such as systolic and diastolic blood pressure and proteinuria. These direct correlations may be a consequence of endothelial dysfunction, on which hypertension and proteinuria probably depend. It has been suggested that an increase in circulating NGAL may be a consequence of leukocyte derived inflammatory activity and endothelial activation, and both of these pathophysiological aspects are involved in HDP. ${ }^{19}$ This would fit with inflammatory and endothelial damage as the central cause in the pathophysiology of preeclampsia, and this can be present in both early and late onset preeclampsia. ${ }^{15}$

In our study, it was observed that $23.07 \%$ of patients in the severe preeclampsia group and $20 \%$ of patients in the eclampsia group had 24 hour urine output less than $400 \mathrm{~mL}$. The mean serum NGAL in these patients was $10593.80 \pm$ $25200 \mathrm{pg} / \mathrm{mL}$, whereas the mean serum NGAL in patients with urine output more than $400 \mathrm{~mL} / 24$ hours was $5545.35 \pm$ $5457.6 \mathrm{pg} / \mathrm{mL}$. There was a significant difference between the two groups $(P=0.001)$. There are no studies demonstrating 


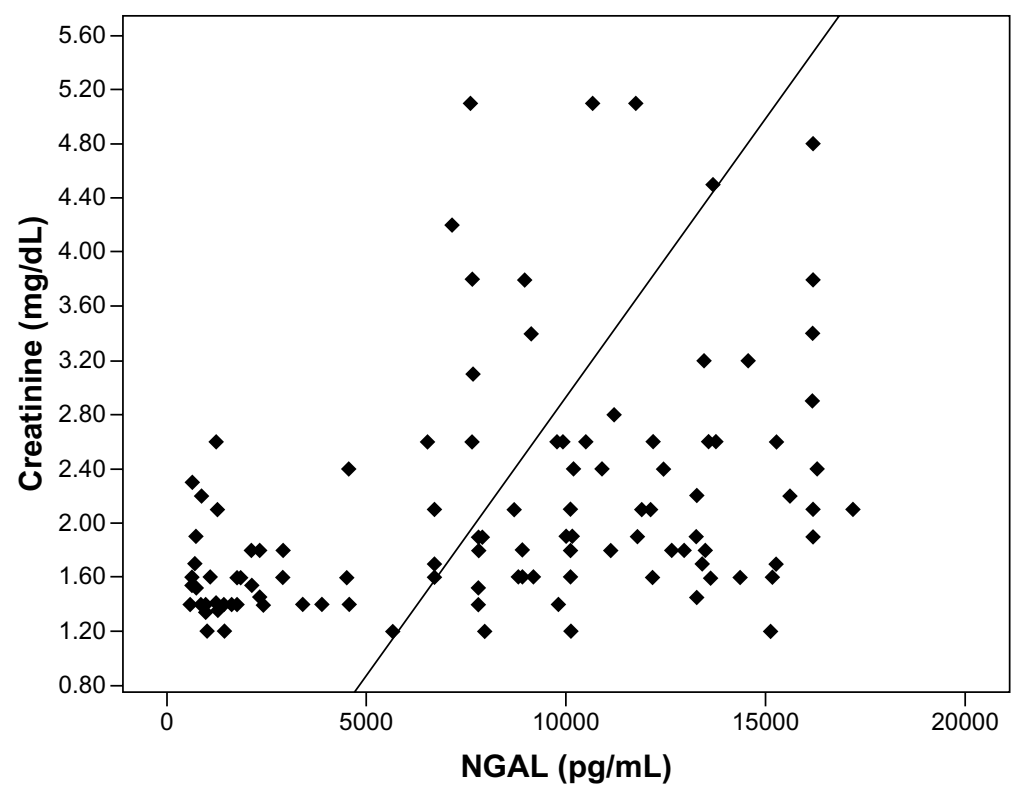

$$
\begin{aligned}
& y=1.56+0.00007 \\
& r=0.4(P<0.01)
\end{aligned}
$$

Notes: There is a significant positive correlation between values of neutrophil gelatinase-associated lipocalin and creatinine. Increases in the values of neutrophil gelatinaseassociated lipocalin result in increases in the values of creatinine.

Abbreviation: NGAL, neutrophil gelatinase-associated lipocalin.

direct correlation between serum NGAL with 24 hour urine output in preeclampsia and eclampsia.

In HPD, usually we measure disease severity with proteinuria but there is no method of measuring proteinuria $100 \%$ reliably. Secondly, when 24 hour protein excretion exceeds $300 \mathrm{mg} /$ day, fetal outcomes are generally worse than in pregnancies where there is less proteinuria. ${ }^{20}$ Thus, we studied the novel marker NGAL in HPD to understand the association between proteinuria and NGAL. In our study, not only was an association between proteinuria with disease severity found, but also a positive association was observed between serum NGAL and proteinuria. Similarly, another study has reported positive correlation between serum NGAL and proteinuria $(r \sim 0.59)$ in patients with preeclampsia. ${ }^{21}$

A study ${ }^{22}$ has reported there was a positive correlation between NGAL and proteinuria in pregnancies complicated by

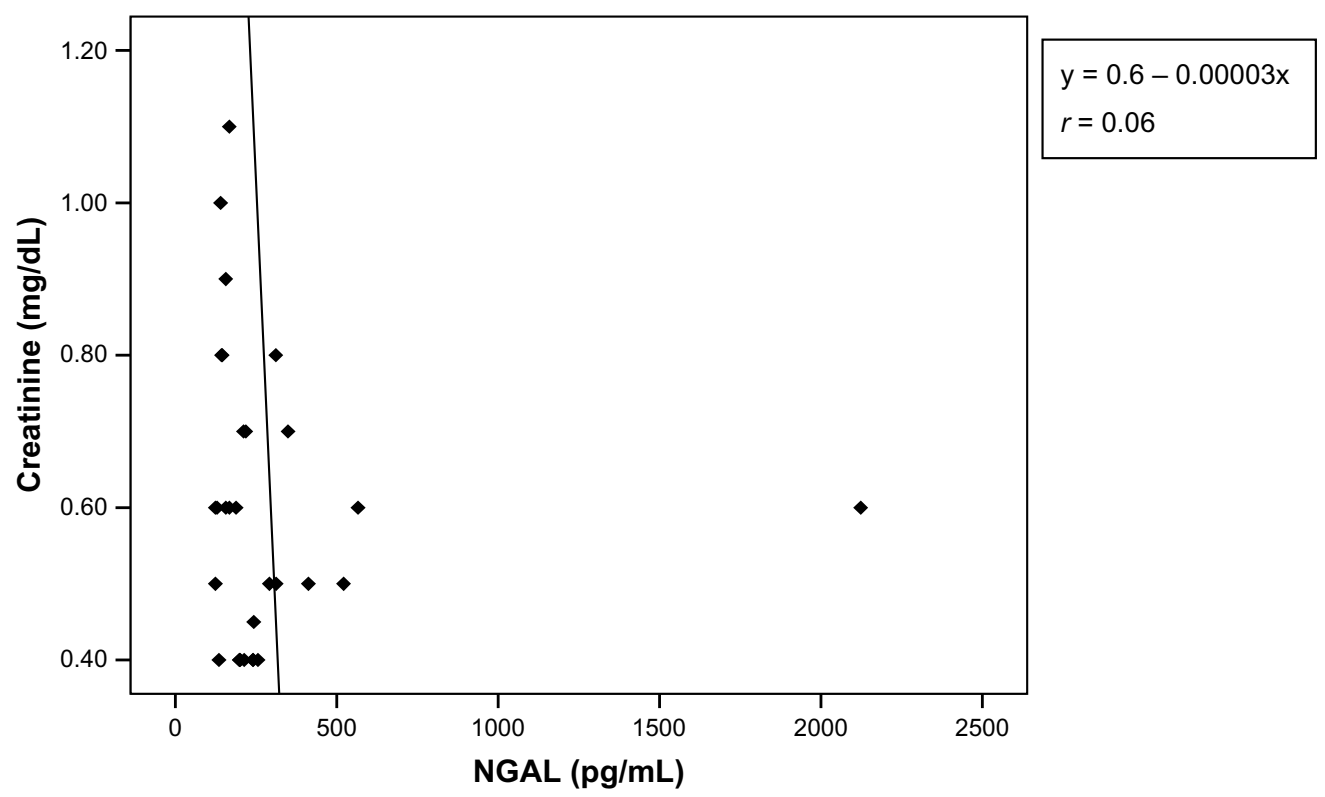

Figure 4 Scatter plot of serum creatinine versus neutrophil gelatinase-associated lipocalin (control).

Notes: There is a negative correlation between values of neutrophil gelatinase-associated lipocalin and serum creatinine. Increases in the values of NGAL result in decreases in the values of serum creatinine.

Abbreviation: NGAL, neutrophil gelatinase-associated lipocalin. 
preclampsia, this might be confirmed the association between NGAL and renal dysfunction. ${ }^{22}$ This data may be in agreement with recent evidence in the literature on urinary and plasma NGAL as an early marker of renal damage. Therefore, we conclude that endothelial damage may be present from the first trimester of pregnancies only, which were subsequently complicated by preeclampsia. These data may be of interest because our HDP cases were almost all late onset; consequently, it seems that also in milder types of preeclampsia there is early endothelial dysfunction that reaches a peak in the second half of pregnancy.

In our study, a significant difference was observed between the mean value of serum NGAL in the control group and AKI stages I and II and acute renal failure and an increasing trend was observed when moving from the control group to various grades of AKI. NGAL was found to be significantly correlated with creatinine in the cases with the value of the correlation coefficient being 0.4. In view of the results, NGAL is a sensitive indicator of AKI and it has correlation with various parameters.

\section{Conclusion}

The positive correlation between serum NGAL and systolic blood pressure, diastolic blood pressure, serum creatinine, and proteinuria highlighted in this study may confirm the association between NGAL and renal dysfunction. These direct correlations may be a consequence of endothelial dysfunction on which hypertension and proteinuria probably depend. NGAL was found to be significantly correlated with creatinine in the cases with the value of correlation coefficient being 0.4 . Our study showed that NGAL is a sensitive indicator of AKI and is positively correlated with various parameters.

\section{Disclosure}

The authors report no conflict of interest in this work.

\section{References}

1. Khan KS, Wojdyla D, Say L, Gulmezoglu AM, Van Look PF. WHO analysis of causes of maternal death: a systematic review. Lancet. 2006;367:1066-1074.

2. Montan S, Sjoberg NO, Svenningsen N. Hypertension in pregnancy - fetal and infant outcome. A cohort study. Clin Exp Hypertens B. 1987;6:337-348.

3. Xu S, Venge P. Lipocalins as biochemical markers of disease. Biochem Biophys Acta. 2000;1482(1-2):298-307.
4. Matthys LA, Coppage KH, Lambers DS, Barton JR, Sibai BM. Delayed postpartum preeclampsia: an experience of 151 cases. Am J Obstet Gynecol. 2004;190(5):1464-1466.

5. Bosch JP. Renal reserve: a functional view of glomerular filteration rate. Semin Nephrol. 1995;15(5):381-385.

6. Mishra J, Mori K, Ma Q, Kelly C, Barasch J, Devarajan P. Neutrophil gelatinase-associated lipocalin: a novel early urinary biomarker for cisplatin nephrotoxicity. Am J Nephrol. 2004;24:307-315.

7. Mishra J, Dent C, Tarabishi R, et al. Neutrophil gelatinase-associated lipocalin (NGAL) as a biomarker for acute renal injury. Lancet. 2005; 365(9466):1231-1238.

8. Report of the National High Blood Pressure Education Program Working Group on High Blood Pressure in Pregnancy. Am J Obstet Gynecol. 2000;183(1):S1-S22.

9. Clinical practice guideline on acute kidney injury [webpage on the Internet]. Brussels: Kidney Disease: Improving Global Outcomes; 2011. Available from: http://www.kdigo.org/clinical_practice_guidelines/pdf/ KDIGO\%20AKI\%20Guideline.pdf. Accessed July 18, 2013.

10. Kumar N, Gupta N, Kishore J. Kuppuswamy's socioeconomic scale: updating income ranges for the year 2012. Indian J Public Health. 2012;56:103-104.

11. Devarajan P. Neutrophil gelatinase-associated lipocalin: a promising biomarker for human acute kidney injury. Biomark Med. 2010;4: 265-280.

12. Workeneh BT. Acute renal failure. Available from: http://emedicine. medscape.com/article/243492-overview. Accessed July 18, 2013.

13. Nalini Arora, Kirti Mahajan Narayan Jana, Abhijit Taraphder. Pregnancy related Acute renal failure in eastern India. International Journal of Gynaecology and Obstetrics. 2010;111:213-216.

14. SivaKumar V, Sivaramakrishna G, Sainaresh VV. "Pregnancy related acute renal failure: a ten year experience". Saudi Journal of Kidney Disease and Transplantation. 2011;22(2):352-353.

15. D’Anna R, Baviera G, Giordano D, Todarello G, Corrado F, Buemi M. Second trimester neutrophil gelatinase-associated lipocalin as a potential prediagnostic marker of preeclampsia. Acta Obstet Gynecol Scand. 2008;87:1370-1373.

16. Sibai BM, Mercer B, Sarinoglu C. Severe preeclampsia in the second trimester: recurrence risk and long-term prognosis. Am JObset Gynecol. 1991;165(5 Pt 1):1408-1412.

17. Barton JR, O’Brien JM, Bergauer NK, Jacques DL, Sibai BM. Mild gestational hypertension remote from term: progression and outcome. Am J Obstet Gynecol. 2001;184(5):979-983.

18. Sibai BM, Sarinoglu C, Mercer BM. Eclampsia. VII. Pregnancy outcome after eclampsia and long-term prognosis. Am JObstet Gynecol. 1992;166(6 Pt 1):1757-1761; discussion 1761-1763.

19. Anwaar I, Gottsater A, Ohlsson K, Mattiasson I. Increasing levels of leukocyte-derived inflammatory mediators in plasma and cAMP in platelets during follow-up after cerebral ischemia. Cerebrovas Dis. 1998;8:310-317.

20. Homer CS, Brown MA, Mangos G, Davis GK. Non-proteinuric pre-eclampsia: a novel risk indicator in women with gestational hypertension. J Hypertens. 2008;26:295-302.

21. D’Anna R, Baviera G, Giordano D, et al. Neutrophil gelatinaseassociated lipocalin serum evaluation through normal pregnancy and in pregnancies complicated by preeclampsia. Acta Obstet Gynecol Scand. 2010;89:275-278.

22. Roberts JM, Gammil HS. Preeclampsia: Recent insights. Hypertension. 2005;46:1263-1269.

\section{Publish your work in this journal}

The International Journal of Nephrology and Renovascular Disease is an international, peer-reviewed open-access journal focusing on the pathophysiology of the kidney and vascular supply. Epidemiology, screening, diagnosis, and treatment interventions are covered as well as basic science, biochemical and immunological studies. The journal welcomes original research, clinical studies, reviews \& evaluations, expert opinion and commentary, case reports and extended reports. The manuscript management system is completely online and includes a very quick and fair peerreview system, which is all easy to use. Visit http://www.dovepress.com/ testimonials.php to read real quotes from published authors. 